Journal of Computational Acoustics (C) IMACS
Electronic version of an article published as Journal of Computational Acoustics 2012 Volume 20 Issue. 2

Article DOI: $10.1142 / S 0218396 X 12400073$

(C) copyright World Scientific Publishing Company http://www.worldscientific.com/worldscinet/jca

\title{
Low- to mid-frequency scattering from elastic objects on a sand sea floor: Simulation of frequency and aspect dependent structural echoes
}

\author{
Mario Zampolli* \\ Sonar Group, TNO, \\ The Hague, 2597 AK, The Netherlands \\ mario.zampolli@tno.nl \\ Aubrey L. Espana, Kevin L. Williams, Steven G. Kargl, Eric I. Thorsos \\ Applied Physics Laboratory, University of Washington, \\ Seattle, WA 98105, USA \\ Joseph L. Lopes, Jermaine L. Kennedy \\ Naval Surface Warfare Center, Panama City Division, \\ Panama City, FL 3240\%, USA \\ Philip L. Marston \\ Department of Physics and Astronomy, Washington State University, \\ Pullman, WA 99164, USA \\ Received (Day Month Year) \\ Revised (Day Month Year)
}

\begin{abstract}
The scattering from roughly meter-sized targets, such as pipes, cylinders and unexploded ordnance (UXO) shells in the $1-30 \mathrm{kHz}$ frequency band is studied by numerical simulations and compared to experimental results. The numerical tool used to compute the frequency and aspect dependent target strength is a hybrid model, consisting of a local finite-element model for the vicinity of the target, based on the decomposition of the three-dimensional scattering problem for axially symmetric objects into a series of independent two-dimensional problems, and a propagation model based on the wavenumber spectral integral representation of the Green's functions for layered media.
\end{abstract}

Keywords: finite element; hybrid model; target scattering.

\section{Introduction}

The scattering from targets at low- to mid-frequencies, typically below $50 \mathrm{kHz}$ for targets of size on the order of a meter, includes significant elastic scattering effects. The superposition of these effects with the geometric scattering returns from the target, makes it difficult to detect and classify objects in sonar images at such frequencies with image based analogies. However, low- to mid- frequency elastic echo enhancements produce highlights in the target

\footnotetext{
*Corresponding author
} 
response, which can be found in images of the target strength $(T S)$ as a function of aspect angle and frequency. Such images can be thought of as the "acoustic fingerprints" of the targets, and are the object of current investigations on target detection and classification through the elastic response. The images of $T S$ versus aspect angle and frequency are also commonly referred to as "acoustic color plots," or "acoustic templates." The latter terminology is used in what follows below.

From the computational point of view, the study of scattering from elastic structures surrounded by one or more fluids, such as water or for example water saturated sediments, which can be represented in a first approximation as fluids, is a challenging problem. For many targets of interest, the shape exhibits either axial or plane symmetry. However, the internal structure of the target often consists of a variety of compartments, filled either with fluids or solids. The tools of choice for modeling geometrically complex objects of this kind are typically finite-element (FE) methods. Fully three-dimensional approaches require the use of large parallel computers, since the number of degrees-of-freedom for the target plus a minimal amount of surrounding fluid would be on the order of a few million. At the present time, all techniques available for such kinds of problems are frequency domain techniques, for which one needs to solve the FE problem over the full bandwidth of interest, with frequency increments sufficiently small to allow for the capturing of all relevant interference patterns in the acoustic template, and of all relevant time-domain signal features obtained by the inverse Fourier transform. The time-domain results are of importance for comparison with synthetic aperture sonar (SAS) data.

In this paper, a structural acoustic FE model capable of computing the scattering from axially symmetric objects surrounded by a fluid, and insonified by a non-symmetric incident field, such as for example a plane wave, is used to obtain acoustic templates for a number of targets in a realistic experimental setting. The approach makes it possible to run models on commonly available single or multi-core desktop workstations. The targets studied below are (i) an aluminum pipe of 2:1 aspect ratio and (ii) two variants of an unexploded ordnance (UXO) shell shape made of solid aluminum. A 2:1 aspect ratio solid aluminum cylinder, ${ }^{1}$ is used to illustrate the usefulness of elastic displacement plots on the target boundary, through which the wave types that characterize some of the features seen in the acoustic templates can be visualized.

The model is briefly reviewed in Section 2, and modeling results are compared to experimental data acquired in a controlled setting in a test pond in Section 3. The target surface displacements, associated with some of the elastic effects visible in the acoustic template plots of a cylindrical target, are visualized and discussed briefly in Section 4. Section 5 contains the conclusions and an outlook on open issues and future work.

\section{Model}

Throughout the entire paper, the time-dependence $\exp (i \omega t)$, with $i$ denoting the imaginary number, $\omega=2 \pi f$ the angular frequency and $t$ being time, is factored out of the equations. The pressure in the fluid, $p$, is described by the Helmholtz equation, and the elastic displace- 
ments by the time-harmonic elastic wave equation. ${ }^{2}$ An axisymmetric elastic target, which can consist for example of a shell and may contain internal fluid and/or solid partitions, is considered. The axis of symmetry of the target coincides with the $z$ axis of a cylindrical coordinate system $(r, z, \theta)$. In general, any incident acoustic pressure field, $p^{\text {inc }}$, which is not necessarily constrained to be symmetric, can be decomposed into a series of azimuthal Fourier modes:

$$
p^{\mathrm{inc}}(r, z, \theta)=\sum_{m=-\infty}^{\infty} p_{m}^{\mathrm{inc}}(r, z) \exp (i m \theta) .
$$

In the case where a plane wave is incident, the sum in Eq. (1) is only over non-negative modal orders, $m \geq 0$, and the azimuthal Fourier coefficients become

$$
p_{m}^{\text {inc }}(r, z)=\epsilon_{m} i^{m} \exp (i k z \sin \phi) J_{m}(k r \cos \phi)
$$

where $k=\omega / c$ is the wavenumber, $\epsilon_{m}=2$ for positive mode orders, and $\epsilon_{0}=1$, and the exponential in Eq. (1) is replaced by $\cos (m \theta)$. $J_{m}$ is the Bessel function of order $m$. The incidence angle of the plane wave relative to the $r$ axis, is $\phi$, with positive angles corresponding to the positive $z$ direction.

The decomposition into azimuthal Fourier modes is also applied to the dependent variables, i.e., to the scattered pressure, $p^{\text {scat }}=p-p^{\text {inc }}$, and to the elastic displacement components $u, v, w$, in the variational equations for the acoustic and elastic fields. ${ }^{2}$ The coupling between fluid and solid is governed by the conditions of continuity of normal displacement, and continuity of normal stress with negative pressure on the wet surface of the target. The integrals over $\theta$ appearing in the variational expressions are easily done analytically. This results in a number of independent 2-D FE problems, one for each azimuthal Fourier mode, from which the $r$ and $z$ dependent azimuthal modal coefficients of the solution are computed. The solution is recovered by superimposing the individual azimuthal modal coefficients multiplied by the appropriate azimuthal Fourier mode. The number of degreesof-freedom (DOF) of each individual 2-D problem is more than one order of magnitude smaller than the number of DOF of the original full 3-D problem, and hence the solution on standard desktop workstations becomes viable even for structural acoustic problems well in the mid-frequency regime. The size of the computational domain is kept small by using perfectly matched layers (PML's) ${ }^{2,3}$ placed in direct contact with the elastic target, or at a small distance (typically much less than one acoustic wavelength) from it.

The pressure at a point $\mathbf{r}_{i}$ outside a surface $S$ enclosing the target is obtained from the FE solution in a post-processing step, using the discrete representation of the Helmholtz integral (often refferred to also as the Helmholtz-Kirchhoff integral): ${ }^{4}$

$$
p\left(\mathbf{r}_{i}\right)=\sum_{j}\left(\frac{\partial G_{i j}}{\partial n_{j}} p_{j}-\rho \omega^{2} G_{i j}\left(u_{n}\right)_{j}\right) d A_{j}
$$

where the index $j$ denotes the point on $S$ where the pressure $p$ and the displacement normal to $S,\left(u_{n}\right)_{j}$, are sampled from the FE solution. The pressure and normal displacement in Eq. 
(3) can be either the total field quantities or the scattered field quantities. In the present paper, the scattered field is of interest. $G_{i j}$ is the Green's function between the point on the surface and $\mathbf{r}_{i}$ for the background medium, ${ }^{4}$ which may be a homogenous infinite fluid, or a layered medium for example, such as a water half-space above a sand half-space. The normal $n_{j}$ is the outward normal on $S$, and $d A_{j}$ is the portion of the area on $S$ associated with the $j$-th point. The density of the background medium, $\rho$, can be position dependent.

The quantity of interest derived from the hybrid local FE - propagation model described above, is the (absolute) target strength, $T S$, here defined as:

$$
T S=20 \log _{10}\left(\frac{R}{1 \mathrm{~m}}\left|\frac{p^{\mathrm{scat}}\left(\mathbf{r}_{i}\right)}{p^{\text {inc }}}\right|\right)(\text { in } \mathrm{dB})
$$

where $R$ is the distance from the target center to the receiver point $\mathbf{r}_{i}$ and $\left|p^{\text {inc }}\right|$ is the amplitude of the acoustic field incident on the target at the location in the fluid corresponding to the target center.

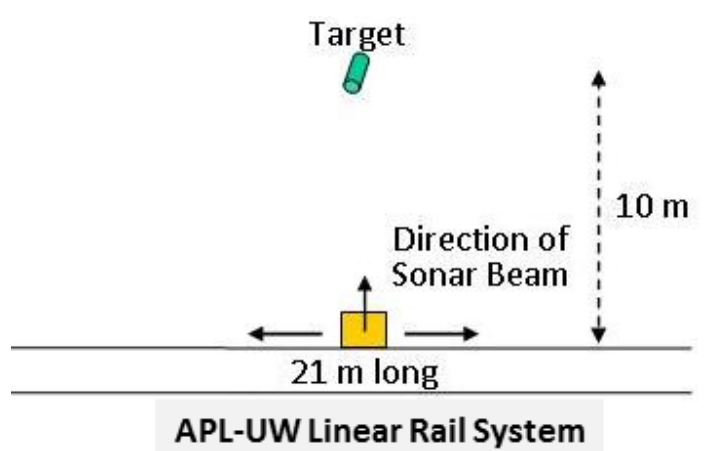

Fig. 1. Geometry of the experimental setup

The experimental setup is shown in Fig. 1. Source and receiver(s) are mounted on a tower, which slides along a straight rail that is placed on a sandy bottom. The target(s) are deployed proud on the sand, at a distance of $10 \mathrm{~m}$ from the rail. By sliding the tower along the rail, it is possible to obtain different source/receiver-target angles, henceforth referred to as aspect angles. Furthermore, it is possible to extend the range of aspect angles by manually rotating the target relative to the rail.

Figure 2 shows a target in relation to the tower at a given position. The $z$ axis coincides with the axis of symmetry of the target in the FE model, and the $x$ axis is parallel to the sea floor. The field incident on the target can be decomposed into a direct incident field, corresponding to the thick dashed arrow in Fig. 2, and a boundary reflected incident field. The direct incident field and the boundary reflected incident field are shown in Fig. 3(a) from a viewing angle in which the axis of the cylinder points into the page (i.e., from the back of the cylinder).

The scattered field arriving at the receiver on the tower is obtained by summing coherently the contributions from the field scattered by the cylinder, idealized as being in an 


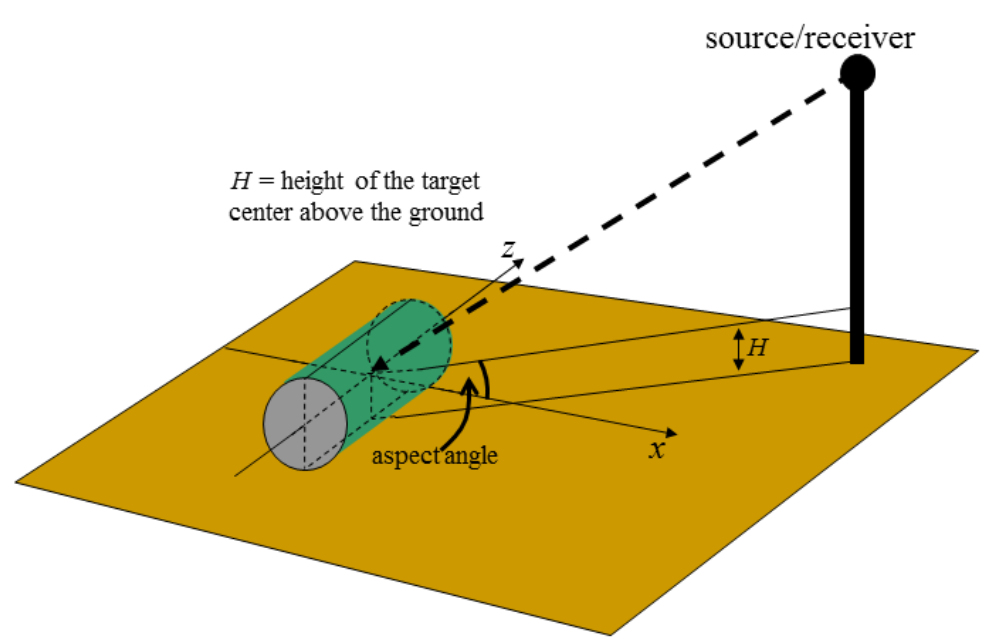

Fig. 2. Target cylinder (or equivalently surface S surrounding the target), relative to the seabed and the source/receiver. The definition of the aspect angle is shown. The dashed arrow denotes the direction of the incident field. The tower, represented by the thick line on which the source/receiver sits, is perpendicular to the seabed (i.e., the ground). Thin dashed lines are inside the target cylinder.

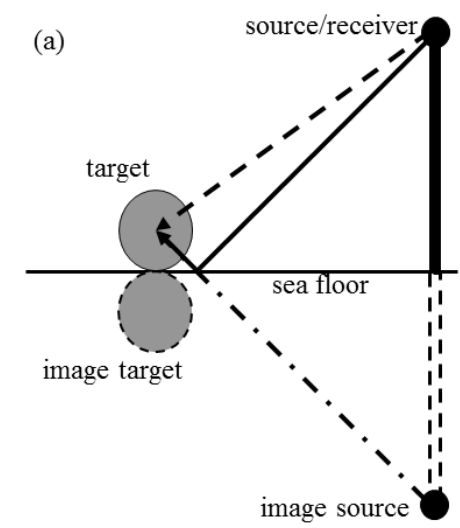

(b)

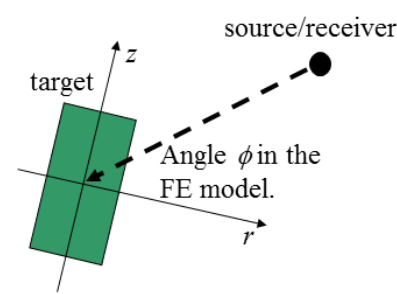

The $r-z$ plane contains the

source point.

Fig. 3. (a) View of the target of Fig. 2 from the back, with the $z$ axis pointing into the page. The incident field consists of the direct source-target incident field and the boundary reflected incident field (solid arrow). The aspect angle of the 3-D Fig. 2 must be mapped onto the aspect angle $\phi$ of the FE model (b). The same must be done also for the image source, to account for the boundary reflected incident field.

infinite fluid, ensonified by the direct and the boundary reflected incident wave of Fig. 3(a). In each case, the target scattered field is propagated back to the receiver by the discrete Helmholtz integral of Eq. (3), in which the Green's function for the two-layered background medium is given by the coherent superposition of a free-field point source located on the target surface, $S$, and an image point source located on the image target in Fig. 3(a). The image point source field is multiplied by the plane wave reflection coefficient for a plane wave incident on the interface at an angle equal to the angle formed by the line connecting 
the image target surface point and the receiver, and the interface. This approximation is consistent with the first order steepest descent evaluation of the wavenumber spectral integral representation of the Green's function for the two layered medium. ${ }^{4}$ For each of the two incident field cases, the aspect angle between the source and the target, and the image source and the target respectively, needs to be mapped into the coordinate system of the FE computation, in which the source, or respectively the image source, is contained in the $r$ - $z$ plane, according to Fig. 3(b). The present approach neglects higher order re-scattering between the target and the surface, as well as impedance discontinuities on the portion of the target surface in contact with the sea floor.

\section{Model/Data Comparisons}

It is necessary to first verify that the free-field finite element results are indeed correct, since these are used to build up the proud simulation using the aforementioned hybrid model. This validation is performed for an aluminum pipe with open ends, $0.6096 \mathrm{~m}$ in length, $0.3048 \mathrm{~m}$ in diameter and with a wall thickness of $0.0095 \mathrm{~m}$. The free-field measurements were conducted at the Acoustic Test Facility (ATF) at the Naval Surface Warfare Center Panama City Division (NSWC PCD). The pipe, source and receiver were suspended midwater column. The source and receiver were located $9 \mathrm{~m}$ away from the pipe, and had a $1 \mathrm{~m}$ separation between them. The source transmitted a $3 \mathrm{~ms}$ linear frequency modulated (LFM) pulse, with a bandwidth from $8-40 \mathrm{kHz}$ and center frequency of $24 \mathrm{kHz}$. The sound scattered in this near backward direction was recorded on a three channel vertical receiver array. The pipe was rotated in one degree steps, from 0 (broadside) to 90 (end-on). The 90 degrees are sufficient to build up a full 180 degree acoustic template due to the symmetry of the pipe. Fig. 4(a) shows the absolute target strength acquired on one channel, as a function of frequency and aspect (or rotation) angle. The finite element simulation for the pipe sampled the pressure and derivatives in the near field of the pipe, approximately 0.5 $\mathrm{mm}$ away from the pipe wall. The full 3-D results were built up using the method described above, namely the mapping of FE domain angles onto the experimental angles, and using the discrete sum representation of the Helmholtz integral. Fig. 4(b) shows the absolute target strength obtained from the FE simulation. There is good agreement between the data and model results. The strongest response from the pipe is observed at broadside orientation, where the scattering is dominated by the specular return from the front face of the pipe. A number of other enhancements are found off-broadside, which are due to the elastic response of the pipe.

With the confidence in the FE model predictions of the acoustic response of the target in the free-field, it is now possible to make comparisons between experiment and model results for the acoustic scattering from targets in contact with a sand sediment. As previously described, the experimental results presented here were taken at NSWC PCD's Facility 383 test pond. Further details of the experiment have been described previously. ${ }^{5}$ Fig. 5 compares the acoustic templates for the aluminum pipe used in the free-field study described above, now in contact with a sand sediment. 

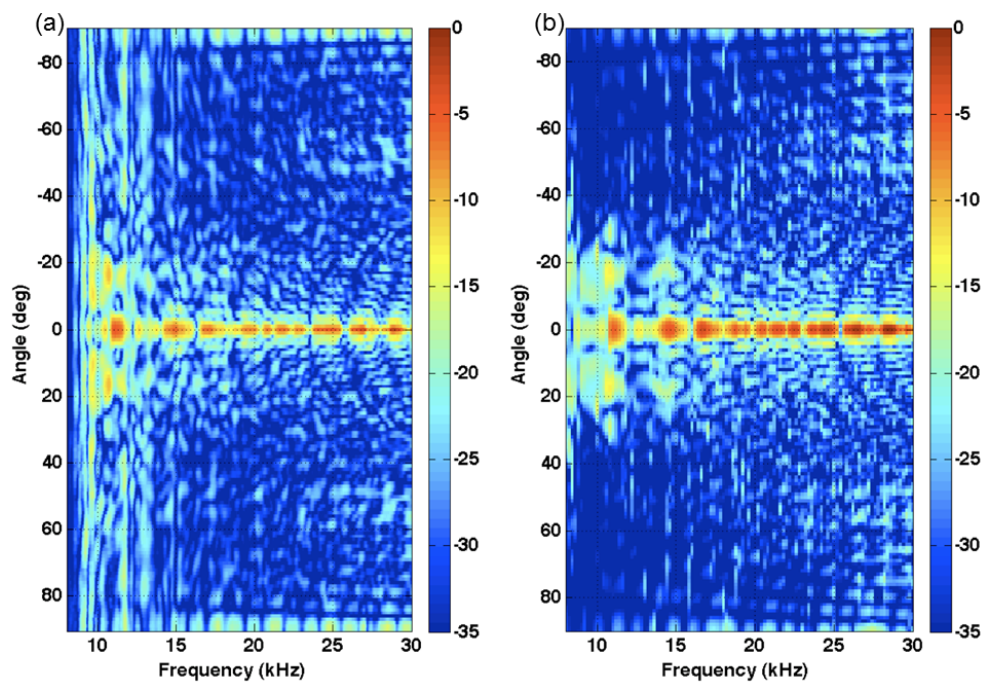

Fig. 4. Data/model comparison of the absolute target strength as a function of frequency and aspect angle for an aluminum pipe suspended in the free-field. (a) shows the experimental results acquired in the NSWC PCD tank experiment, and (b) is the result of the finite element simulation with geometry matching that of the tank experiment.
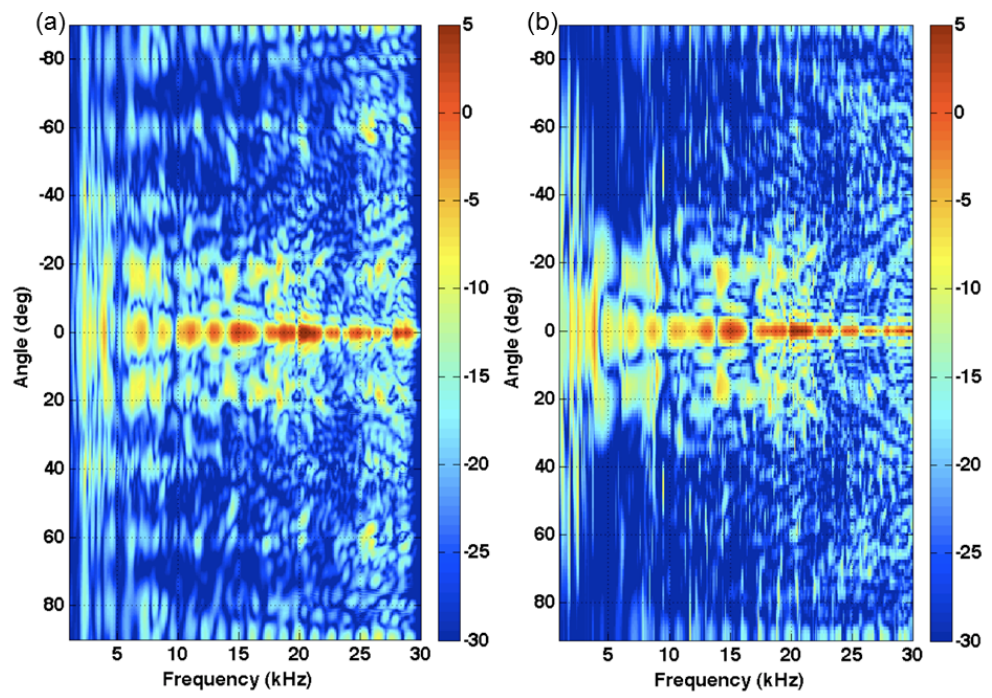

Fig. 5. Data/model comparison of the absolute target strength as a function of frequency and aspect angle for an aluminum pipe proud on a sand sediment. (a) shows the experimental results acquired during the pond experiment (PondEx10), and (b) is the result of the finite element simulation with geometry matching that of the pond experiment. Note the $5 \mathrm{~dB}$ change in scale relative to Fig. 4.

The next target examined is a solid, aluminum replica of an unexploded ordance (UXO) shell. The UXO measures $0.108 \mathrm{~m}$ in diameter and $0.413 \mathrm{~m}$ in length. A composite picture showing the UXO with the corresponding mesh used for the finite element model is given 
in Fig. 6.

The acoustic templates corresponding to the data collected during the pond experiment are shown in Fig. 7(a). Two FE models were created to determine the influence of the ridges and notches, i.e. the fine structure of the UXO body, on the acoustic target template. The first model included the exact shape of the UXO, the results of which are given in Fig. 7 (b). The second model was a slightly more simplified geometry, in which the fine structure in the exterior of the UXO body was "smoothed-out." Examination of Fig. 7 reveals that the overall response of the target is captured well by both models. Not only do the models succeed in accurately representing the bright response at broadside (0 degrees in the figure), in addition to the sharp resonant peaks around 40-60 deg and 5-15 kHz, they also replicate the arcs visible in the data above $15 \mathrm{kHz}$. Closer examination of the templates shown in Fig. 7 and of the TS plots shown in Fig. 8 reveals the effects of the fine structure of the UXO, which are particularly evident in the $20-30 \mathrm{kHz}$ band. The model with ridges agrees more closely with the experimental data, and the differences between the model with ridges and the smooth model can exceed $10 \mathrm{~dB}$ over significant parts of this frequency band.

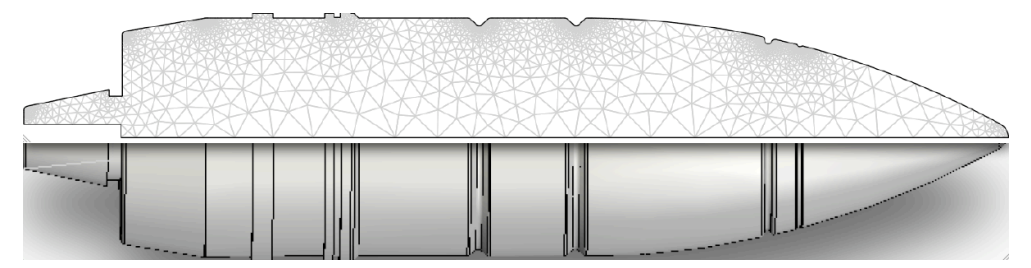

Fig. 6. Schematic of the solid aluminum UXO replica used during the pond experiments and for the FE simulation. The fine structure with ridges and notches can be seen.

\section{Physical Interpretation}

Up to this point, the focus has been on examining the response of targets over a broad angle and frequency range. However, it is possible to gain a better understanding of the dominant elastic effects associated with the scattering features by closer examination of the FE model results. The normal derivative of the scattered pressure, which is proportional to the particle displacements associated with the scattered pressure, along the exterior of the target can be extracted from the FE solution in post-processing. This method is here applied to the case of free-field scattering from a solid aluminum cylinder having a length of $61 \mathrm{~cm}$ and a length to diameter ratio of two, for which the acoustic templates have been studied in detail in an earlier publication. ${ }^{1}$ In order to visualize the purely elastic component of the cylinder response, it is necessary to subtract the rigid, or geometrical, response of the target. To accomplish this, a "semirigid" FE model was run, for which the sound speeds of the aluminum were increased by a factor of 100, while leaving the material density the same. The semirigid results are subtracted from the full elastic FE results, and plots are generated of the normal pressure derivative along the cylinder surface. The subtraction of 


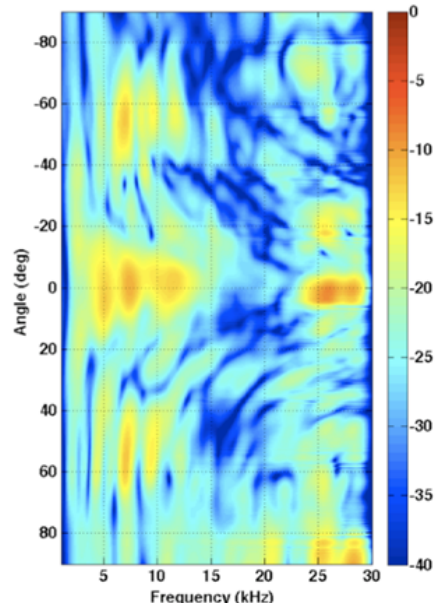

(a)

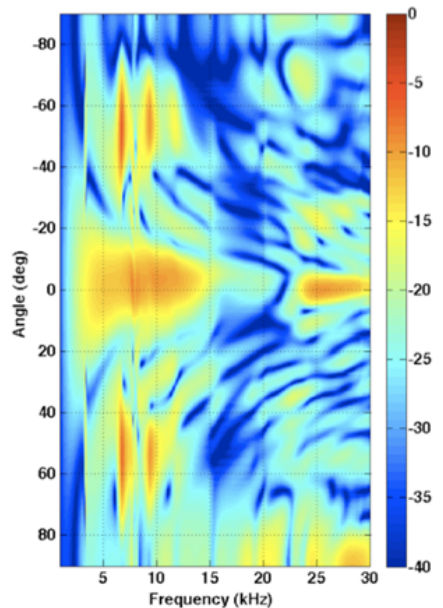

(b)

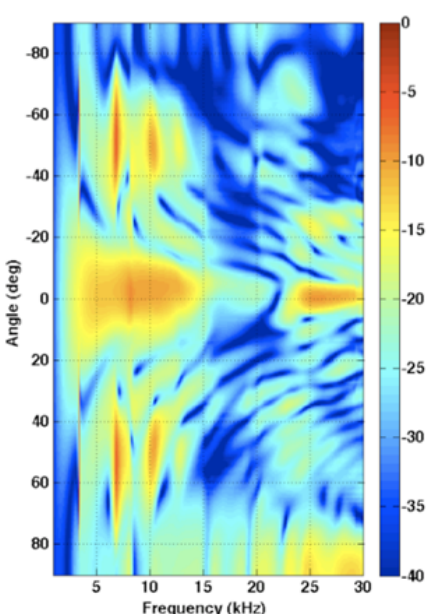

(c)

Fig. 7. Data/model comparison of the absolute target strength as a function of frequency and aspect angle for the aluminum UXO proud on a sand sediment. Nose-on orientation of the UXO corresponds to -90 degrees. (a) shows the experimental results acquired during the pond experiment (PondEx10), (b) is the result of the finite element simulation in which all of the fine structure was included in the model, and (c) is the result of the finite element simulation for the UXO with a smooth exterior.
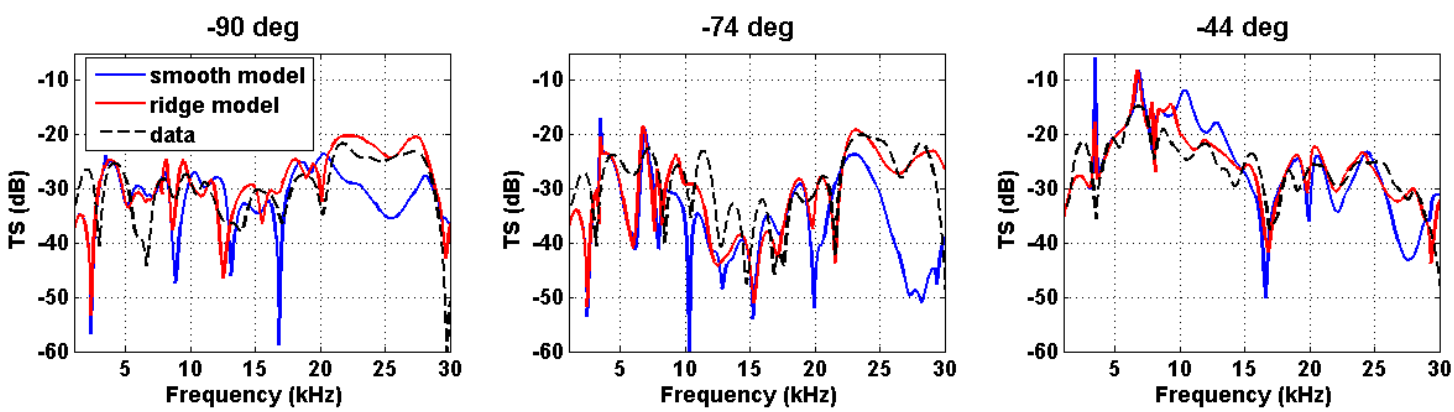

$0 \mathrm{deg}$

$65 \mathrm{deg}$
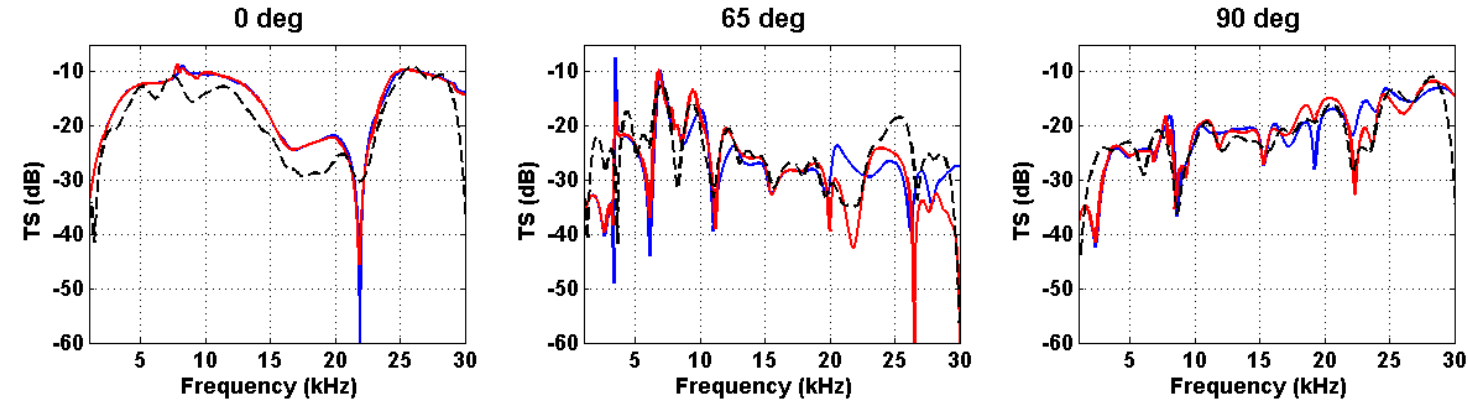

Fig. 8. Data/model comparison of the absolute target strength as a function of frequency, for the proud aluminum UXO, at six different aspect angles. These plots show the TS extracted from Fig. 7(a)-(c) at the angles shown in the plot titles. 
the semirigid result allows to separate non elastic effects, such as the motion of the center of mass, from the purely elastic response. Fig. 9 corresponds to a resonant peak at $16.0 \mathrm{kHz}$ and aspect angle of $32.5 \mathrm{deg}$. The corresponding angle of incidence on the cylinder is 30 $\mathrm{deg}$, and is equal to the Rayleigh wave coupling angle for aluminum in water. ${ }^{1}$ In this case, sound couples to the cylinder and travels down the meridian, ultimately reflecting from the cylinder end and leading to the observed resonant enhancement.

(a) Semirigid

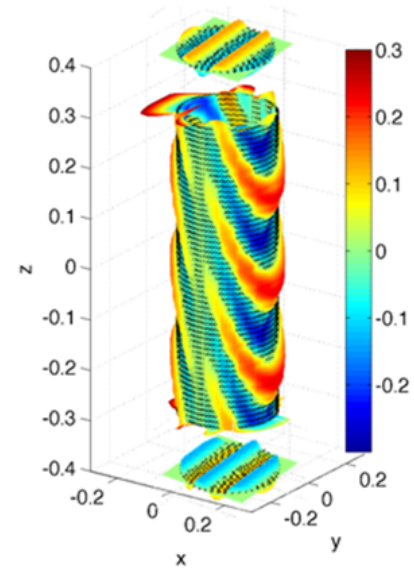

(b)

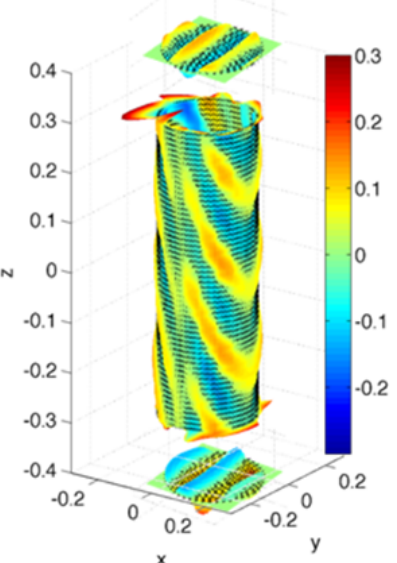

(c) Elastic-Semirigid

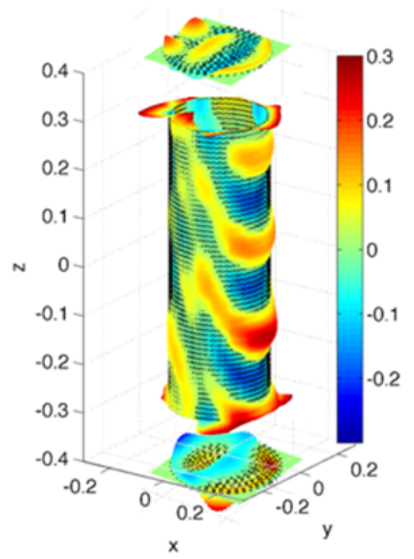

Fig. 9. Colors and surface displacements, proportional to normal pressure derivatives along the cylinder surface for (a) semirigid model, (b) elastic model, and (c) subtraction of the semirigid from the elastic model, resulting from an incoming plane wave at $16.0 \mathrm{kHz}$ with an angle of incidence equal to 30 deg w.r.t. the normal to the cylinder axis. In these coordinates, the incident wave vector has $k_{x}<0, k_{y}=0$ and $k_{z}<0$. The color scales and the displacements are normalized with respect to the maximum displacement in each plot. The top and bottom flat ends of the cylinder are displaced vertically, to improve the visualization of the displacements at the terminations.

Fig. 10 corresponds to a resonant peak at $12.5 \mathrm{kHz}$ and aspect angle of $25 \mathrm{deg}$ (angle of incidence $23 \mathrm{deg}$ ). In this situation, the sound is incident on the cylinder below the Rayleigh wave coupling angle, resulting in an enhancement to the acoustic scattering from helical waves that reflect from the end of the cylinder.

Fig. 11 corresponds to a resonant peak at $22.0 \mathrm{kHz}$ and aspect angle of $64 \mathrm{deg}$ (angle of incidence $57 \mathrm{deg}$ ). This corresponds to the excitation of a face-crossing Rayleigh wave, ${ }^{1,6}$ which can be seen in Fig. 11(c), where the maximum particle displacements are observed near the front end of the cylinder.

\section{Conclusions}

The hybrid model consisting of a FE model for axisymmetric targets with non-symmetric loading and a discrete representation of the Helmholtz integral in which the Green's functions for a layered background medium are employed, is a tool capable of treating experi- 

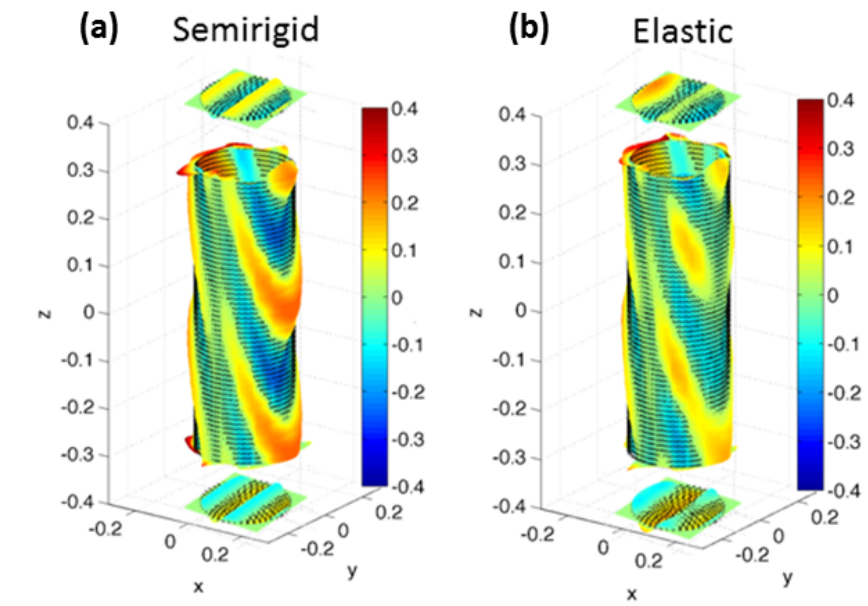

\section{(c) Elastic-Semirigid}

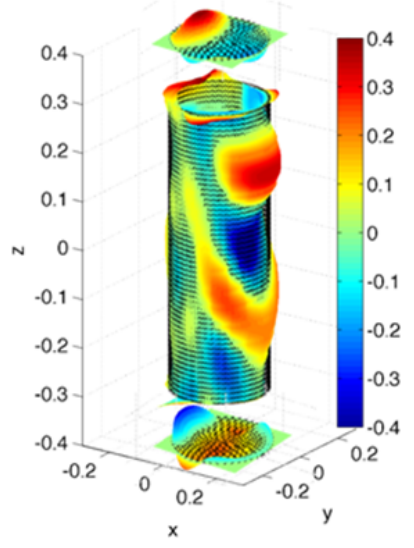

Fig. 10. Normal pressure derivative color and displaced surface plots, along the cylinder surface for (a) semirigid model, (b) elastic model, and (c) subtraction of the semirigid from the elastic model, resulting from an incoming plane wave at $12.5 \mathrm{kHz}$ with an angle of incidence equal to $23 \mathrm{deg}$ w.r.t. the normal to the cylinder axis. In these coordinates, the incident wave vector has $k_{x}<0, k_{y}=0$ and $k_{z}<0$. The color scales and the displacements are normalized with respect to the maximum displacement in each plot.
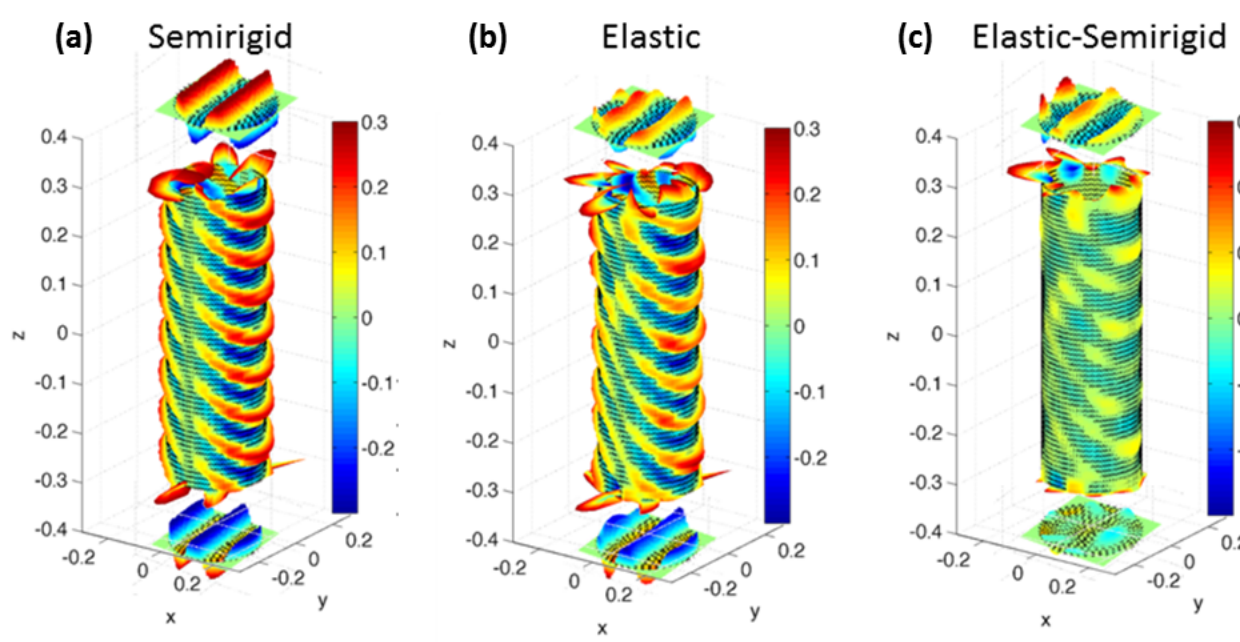

Fig. 11. Normal pressure derivative color and displaced surface plots for (a) semirigid model, (b) elastic model, and (c) subtraction of the semirigid from the elastic model, resulting from an incoming plane wave at $22.0 \mathrm{kHz}$ with an angle of incidence equal to $57 \mathrm{deg}$ w.r.t. the normal to the cylinder axis. In these coordinates, the incident wave vector has $k_{x}<0, k_{y}=0$ and $k_{z}<0$. The color scales and the displacements are normalized with respect to the maximum displacement in each plot.

mentally relevant elastic target scattering problems in the low- to mid- frequency regime. Despite its simplicity, the approach captures most of the relevant physics for targets proud on a water-sand interface, as shown by the agreement between the acoustic templates derived from the computations and the experimental templates. 
The comparison between the smooth UXO and the UXO with ridges, and the cross-check with the corresponding experimental results, shows that the target scattering component of the tool is capable of capturing target details with high fidelity, that determine the typical interference effects exhibited by the acoustic templates. The insight into the physical processes of the target scattering is further aided by the capability of displaying target surface motions in three dimensions, as shown in the examples of Sec. 4.

Two main shortfalls of the model presented here are: (i) the neglection of higher order rescattering between the interface and the target, and (ii) the impossibility of accounting for impedance discontinuities on the wet surface of the target, such as those that would occur for the contact surface between a target and the sediment in a proud or partially buried case. Both these limitations can be studied by comparison to fully 3 -D models. ${ }^{1}$ Buried target scenarios, in which the dominant second order contributions from the boundary reflected incident field response of the proud case are not present, should make it possible to analyze in detail the importance of the higher order rescattering in the target response. The present model is being extended to buried target cases, using Green's functions for sources buried in the sediment. ${ }^{4}$ The comparison between this buried target model, a fully 3-D model and experimental data will make it possible to shed more light onto target-surface rescattering effects, in experimental scenarios with buried targets similar to the ones presented here.

\section{Acknowledgements}

MZ is grateful for the sponsorship from the Department of the Navy Grant N62909-10-17153, issued by the United States Office of Naval Research Global (ONR-G). The contribution of PLM is sponsored through ONR grant N000141010093. The work of APL and NSWC PCD is supported by ONR and by the Strategic Environment Research Development Program (SERDP).

\section{References}

1. K.L. Williams, et al., "Acoustic scattering from a solid aluminum cylinder in contact with a sand sediment: Measurements, modeling, and interpretation," J. Acoust. Soc. Amer. 127 (2010) 3356 -3371 .

2. M. Zampolli, et al., "A computationally efficient finite element model with perfectly matched layers applied to scattering from axially symmetric objects," J. Acoust. Soc. Amer. 122 (2007) $1472-1485$.

3. J.P. Bérenger, "A perfectly matched layer for the absorption of electromagnetic waves," J. Comput. Phys. 114 (1994) 185-200.

4. M. Zampolli, A. Tesei, G. Canepa, O.A. Godin, "Computing the far field scattered or radiated by objects inside layered fluid media using approximate Green's functions," J. Acoust. Soc. Amer. 123 (2008) 4051 - 4058.

5. A.L. Espana, et al., "Measurements and modeling of the acoustic scattering from an aluminum pipe in the free field and in contact with a sand sediment," In Proc. MTS-IEEE Oceans Conf. (2010).

6. K. Gipson, P.L. Marston, "Backscattering enhancements from Rayleigh waves on the flat face of a tilted solid cylinder in water," J. Acoust. Soc. Amer. 107 (2000) 112 - 117. 